\title{
Ewing's sarcoma of femur with metastasis to the breast: a rare case report
}

\begin{abstract}
Ewing's sarcoma/Peripheral primitive neuroectodermal tumors ((EWS)/PNET) are highly malignant small round cell tumors, occurring primarily in limb bones of children and young adults. Usual sites of metastasis are lung, pleura, other bones and occasionally to lymph node, central nervous system and liver. Metastasis to the breast is an extremely rare entity with only few cases reported till date. Nevertheless, it should always be considered as a differential if there is a history of Ewing's sarcoma in a patient presenting with a palpable breast lump. Breast metastasis is indicative of advanced disease with poor prognosis requiring prompt diagnosis and treatment. We hereby present a case of a 22 year old female presenting with a palpable breast lump of 3 months duration, who had a past history of partially treated knee swelling 6 months back. Fine needle aspiration cytology from the breast lump as well as the lower end of femur revealed a similar morphology comprising of dimorphic population of small round cells and larger cells, singly and in rosettes with reticulo-granular chromatin and scant cytoplasm. Based on the immuno-histopathological examination of the excised tissue, the diagnosis of Ewing's sarcoma femur metastasizing to the breast was made.
\end{abstract}

Volume 2 Issue 2 - 2017

\author{
Kafil Akhtar,Alia Ehsan,Asim I Khan, Mubarak \\ Shraim \\ Department of Pathology, Aligarh Muslim University, India
}

Correspondence: Kafil Akhtar, Department of Pathology, Jawaharlal Nehru Medical College, Aligarh Muslim University, Aligarh (UP), India, Email drkafilakhtar@gmail.com

Received: October 14, 2016 | Published: March 22, 2017

Keywords: breast, cytology, ewing's sarcoma, histopathology, metastasis

\section{Introduction}

Ewing's sarcoma/Primitive neuroectodermal tumors (EWS/PNET) comprise a group of rare malignant and aggressive tumors, known to originate from migration of embryonic cells of the neural crest. They characterstically display small round cell morphology and variable neuroectodermal differentiation. These tumors have a predilection for long bones, pelvis, and ribs, occuring commonly in the children and adolescents. ${ }^{1}$ Although extraskeletal involvement of soft tissues either primary or metastatic is quite rare; few cases involving the lung, pleura and lymph node have been reported. ${ }^{2}$ Extra-mammary metastasis to the breast is quite uncommon with prevalence ranging from 1.7 to $6.6 \%$ and is an indicator of disseminated disease with a poor prognosis. ${ }^{3,4}$ Lymphoma, malignant melanoma, rhabdomyosarcoma and lung carcinoma are common tumors that metastasize to the breast. Sarcomas and more specifically Ewing's sarcoma are extremely rare source of origin of breast metastasis with only few cases reported in the literature till date.,

Ancillary techniques are necessary for accurate diagnosis and typing of malignant tumors of breast, due to variation in their prognosis and ways of management. Owing to their usual superficial and accessible locations, fine needle aspiration cytology is usually an important screening tool to evaluate the lesion as benign or malignant and if malignant whether primary or metastasis. However, cytology and histopathological biopsy along with immunohistochemical examination forms the mainstay of diagnosis. Moreover, highlighting the characteristic transcript $t(11,22)$ at molecular level is necessary for a confirmatory diagnosis. ${ }^{6}$ This rare case report shows the importance of a keen cytological and histopathological examination in early diagnosis of metastatic palpable breast lump from a primary Ewing's sarcoma of femur to assure prompt treatment and avoid any unnecessary radical operation.

\section{Case summary}

A 22 year old female presented to the surgical OPD with a gradually increasing painful lump in the right breast since the past 3 months. She also complained of low grade fever with loss of weight and appetite for 5-6months duration. There was no history of cough, chest pain, any loco-regional trauma or any significant family history. She also complained of gradually increasing pain in left knee since 6 months with decreased mobility. On examination, the patient was average built with adequate nutrition. Systemic examination was apparently normal with presence of mild pallor. Breast examination revealed a firm, irregular, non-tender and mobile lump of size $3 \mathrm{~cm} \times 2 \mathrm{~cm}$ in upper outer quadrant of right breast without any skin, lymph node or deep structural involvement. A doppler ultrasound was suggestive of malignancy with BIRADS grade IVa.

Local examination of left knee joint showed an ill-defined firm swelling and tenderness with decreased mobility but absence of any redness or ulceration. X-ray of left knee joint showed an ill-defined lytic lesion with mild periosteal reaction in distal femur along with pathological fracture. Magnetic resonance imaging on both T1 and T2 weighted signals showed an ill-defined mass of increased heterogenesity infiltrating into adjacent tissue. On the basis of which a differential of Ewing's sarcoma and chronic osteomyelitis was given. A fine needle aspiration cytology from both the sites of lesion was performed which showed strikingly similar cytological features of individually scattered as well as cohesive clusters of dual population of cells, comprising of small round hyper chromatic cells with scant cytoplasm and large atypical round to polygonal cells with increased nuclear-cytoplasmic ratio, coarse granular chromatin, indistinct nucleoli and scant cytoplasm. Occasional foci of rosette formation and nuclear moulding were also seen Figure 1.

Based on clinic-pathological findings a diagnosis of small blue round cell tumor probably Ewing's sarcoma of left distal femur with metastasis in right breast was made. For confirmation, an incisional biopsy from both the sites was performed which showed tumor areas comprising of sheets of cells, with predominantly small round cells with hyper chromatic nuclei, scant clear cytoplasm and indistinct cell 
membrane along with few relatively larger atypical cells with reticlogranular chromatin. Evidence of rosette formation was seen at places. Immunohistochemistry showed CD-99 positive tumor cells (Figure 2). Keeping in mind the aggressive nature and prognosis of the tumor, six cycles of combination chemotherapy was administered with Vincristine $50 \mathrm{mg} / \mathrm{m}^{2}$, Adriamycin $25 \mathrm{mg} / \mathrm{m}^{2}$ and Cisplatinum $50 \mathrm{mg} /$ $\mathrm{m}^{2}$ and $50 \mathrm{~Gy}$ of Cobalt-60 teletherapy. After 6 months of follow up, our patient is doing well (Figure 3).

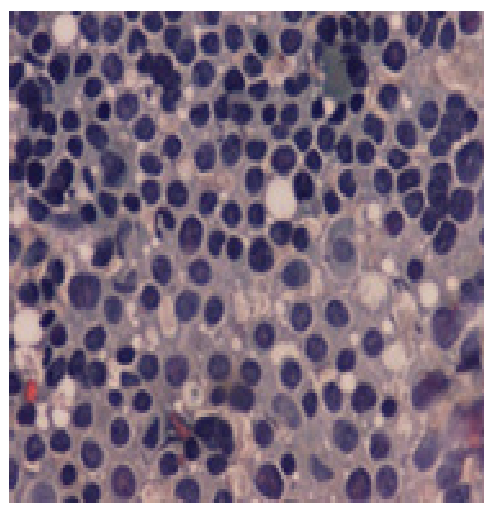

Figure I FNAC showed scattered as well as cohesive clusters of dual population of cells, comprising of small round hyperchromatic cells with scant cytoplasm and large atypical round to polygonal cells with increased nuclearcytoplasmic ratio, coarse granular chromatin, indistinct nucleoli and scant cytoplasm with occasional foci of rosette formation and nuclear moulding. Haematoxylin and eosin $x$ 40X.

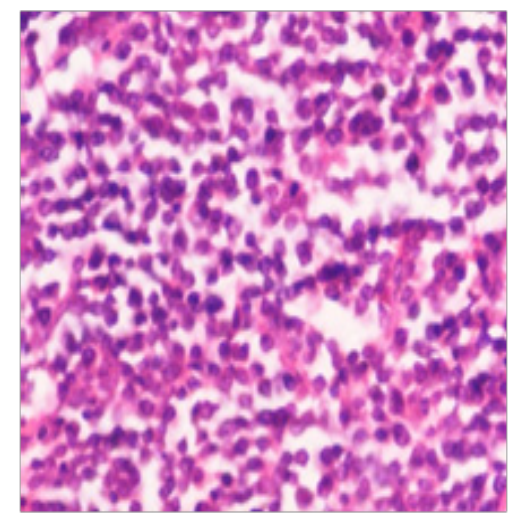

Figure 2 Tumor areas showed sheets of predominantly small round cells with hyperchromatic nuclei, scant clear cytoplasm and indistinct cell membrane along with few relatively larger atypical cells with reticlo-granular chromatin with evidence of rosette formation at places. Haematoxylin and eosin $x 40 \mathrm{X}$

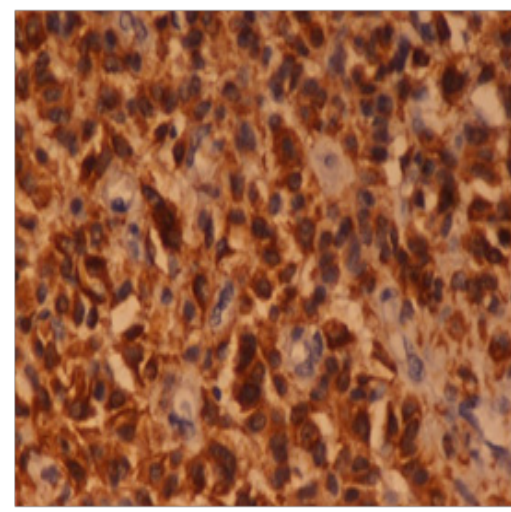

Figure 3 Immunohistochemistry showed CD-99 positive tumor cells. Immunostain CD99 x 40X

\section{Discussion}

Majority of breast malignancies are carcinomas, whether primary or secondary with sarcomas representing less than $1 \% .{ }^{1}$ Metastases to the breast is an unusual entity that may be detected during treatment for a known malignancy, or can rarely be the first manifestation of a malignant disease. They usually presents as a solitary (85\%), firm to hard, mobile, palpable breast mass with a characteristic rapid growth rate. The upper outer quadrant is most commonly involved with a slight left breast predominance $(66 \%))^{2,3}$ The metastatic deposits present clinically as well-defined, mobile and rapidly enlarging masses with absence of pain and/or discharge. ${ }^{7}$ Breast metastasis usually occurs by blood-borne and lymphatic route. Lymphatic metastases are usually found in the medial portion of the breast, whereby the skin of the breast becomes diffusely thick and the breast parenchyma becomes denser with many irregular masses on mammography. Blood-borne metastases are bilateral, with well-defined and spherical metastatic masses. ${ }^{7}$ Melanoma is the most common source of bloodborne metastases into the breast, followed by lung carcinoma and lymphoma. ${ }^{2}$ Primary carcinomas from the stomach, prostate, ovaries, kidneys, cervix, thyroid and the skin are also known to metastasize to the breast. ${ }^{8,9}$

Although any malignancy may metastasize to the breast, but Ewing's sarcoma are extremely rare group as a primary tumor. Firstly reported by Leonardo et al., ${ }^{3}$ there are very few documented cases of skeletal Ewing's sarcoma metastases to breast in the literature. ${ }^{3}$ In approximately $30 \%$ of patients, metastasis to the breast is the first sign of malignancy, and time from initial diagnosis to metastasis to the breast varies between 1 month to 15 years. ${ }^{10}$ Ewing's sarcoma/ Peripheral primitive neuroectodermal tumors ((EWS)/PNET) are small round cell tumors, occurring primarily in bone and soft tissues of the limbs and arise from neuroectodermal elements that probably develop from migrating embryonic cells of the neural crest. ${ }^{6,11}$ This group of tumors is characterized by the presence of the typical translocation $(11 ; 22)(\mathrm{q} 24 ; \mathrm{q} 12)$ and the expression of CD99 antigen (MIC2) on immunohistochemistry. ${ }^{12}$ Children and young adolescents are most commonly affected. ${ }^{6}$

Ewing's sarcoma usually metastasizes to lung, pleura and other bones. Lymph nodes, CNS, and liver may be occasionally involved. The metastasis of Ewing's sarcoma into breast tissue is extremely rare with only one case report being reported in literature. ${ }^{12}$ Metastatic cancer in the breast is often discovered as a superficial solitary mass in the upper outer quadrant. ${ }^{6}$ These metastases manifest clinically as well-defined, mobile and rapidly enlarging masses. ${ }^{6,7}$ The metastasis of extra mammary malignancies into the breast is very unusual. Lymphoma/leukemia, malignant melanoma and carcinoma originating from the lung, stomach, prostate, ovaries, kidneys, cervix, mouth, thyroid are the common tumors that metastasize into breast tissue. Sarcomas rarely metastasize to breast. However, a few case reports are available revealing metastasis from fibrosarcoma and myofibrosarcoma to the breast. ${ }^{5-7}$ In approximately $30 \%$ of patients, metastasis to the breast is the first sign of malignancy, and time from initial diagnosis to metastasis to the breast varies between 1 month to 15 years. Moreover, some reports emphasize that blood-born metastases to the breast are bilateral but often well-defined rounded masses in contrast to the present case. ${ }^{12,13}$ The histological spectrum of breast masses in children and adolescents is different from that of adults. Imaging findings are useful for performing a diagnosis, but in a patient with a known malignancy, any enlarging breast mass, even one with a benign radiological appearance, should be investigated with a biopsy. 
The bony lesion by a plain radiography usually shows a destructive lytic tumor with permeative margins extending into the surrounding soft tissues. ${ }^{11}$ MRI is the imaging modality of choice because of its high-contrast resolution and ability to define the margins of soft tissue component especially on T2-weighted images. ${ }^{14}$ Similar X-Ray and MRI findings were noticed in our patient. Although the mammographic evaluation of breast mass in such cases is quite non-specific but it is certainly helpful in differentiating primary versus secondary breast malignancy. ${ }^{15}$ The classic mammographic finding is a rounded, well-circumscribed hypoechoic mass with hyper echoic foci and/or posterior enhancement without any spiculation, micro calcification or thickening of the skin. The evaluation of size correlates well with clinical measurements because the metastatic mass does not cause surrounding desmoplastic reaction in adjacent normal breast. ${ }^{4}$ In the present case the size of the breast tumor at presentation was $3 \mathrm{~cm} \times 2 \mathrm{~cm}$, which the doppler showed as a well circumscribed heterogeneous mass (BIRADS grade 4a), suggestive of malignancy.

Fine needle aspiration cytology is usually the next mode of investigation to be done in evaluating the nature of such lesions. Cytologically, features are similar to the diagnosis of malignant small round blue cell tumor, displaying a cellular lesion of monomorphic round cells with scant cytoplasm and reticulogranular chromatin forming occasional rossettes and pseudo-rossettes. ${ }^{16}$ Based on these findings, a differential of Ewing's sarcoma and small round cell variant of osteosarcoma can be made which can be confirmed on biopsy. The histopathological pattern is variable depending on the degree of neuroectodermal differentiation. The tumor is arranged in sheets, lobules or trabeculae. Pseudo-rosettes can be seen. Cells vary from small, round cells with round nuclei, fine chromatin, scant cytoplasm and indistinct cell borders to larger cells with irregular nuclear contours. Mitotic activity is high and necrosis may be present. ${ }^{11,16}$

Immuno-phenotyping is necessary to confirm the diagnosis of EWS/PNET, showing positivity of tumor cells for CD99 (MIC2), vimentin and FLI1 and negative for cytokeratin. ${ }^{12}$ CD99 is a cell surface glycoprotein involved in cell adhesion displaying a membranous staining in the tumor cells and helps in the diagnosis of EWS/PNET with a sensitivity of $84 \%$ to $100 \% .^{12,17}$ Reactivity for vimentin, NSE, CD57 and S100 may suggest a diagnosis but are not pathognomonic. ${ }^{12,17}$ The common differential diagnosis could be primary small cell carcinoma, medullary carcinoma, poorly differentiated ductal or lobular carcinoma, ductal carcinoma with neuroendocrine differentiation, metaplastic carcinoma with neuroectodermal differentiation or secondaries to the breast. ${ }^{18,19}$ Positive staining for CD99 and negative for LCA, CK, EMA, S100, Synaptophysin, Chromogranin and Desmin rules out the possibility of other malignant small round cell tumor. Morphology and immunohistochemical investigation with extensive sampling are compulsory to arrive at a conclusion. ${ }^{17-19}$ However, genotypic analysis by DNA- and RNA-based polymerase chain reaction, Southern blotting and fluorescent in situ hybridization are the only confirmatory tools. ${ }^{20}$

EWS/PNET is an aggressive tumor with a high incidence of local recurrence and distant metastasis. A combination of multiple treatment modalities, including surgery, chemotherapy (vincristine, doxorubicin, cyclophosphamide, ifosfamide, and etoposide) and radiation therapy especially in patients of lung metastases, is indicated in Ewing's sarcoma whether localised or metastatic, although the prognosis is worse in the latter group..$^{18}$

\section{Conclusion}

Ewing's sarcoma is a highly malignant bone tumor predominantly arising in young adults and an extremely rare cause of breast metastasis. Metastatic disease should be taken into consideration in the differential diagnosis of any enlarging breast tumor (even with a reassuringly benign radiological appearance), especially when there is history of extramammary neoplasm. So such a possibility should always be kept in mind in a young patient with a soft tissue swelling and a breast lump simultaneously.

\section{Acknowledgements}

None.

\section{Conflict of interest}

The author declares no conflict of interest.

\section{References}

1. Basma E, Hajar H, Nabil M, Breast Ewing's sarcoma/primitive neuroectodermal tumor: A case report and a review of the literature. Webmed Central Breast. 2012;3(10):37-39.

2. Folpe AL, Goldblum JR, Rubin BP, et al. Morphologic and immunophenotypic diversity in Ewing family tumors: a study of 66 genetically confirmed cases. Am J Surg Pathol. 2005;29(8):1025-1033.

3. Leonardo A, Magali TL, Gwanael F, et al. Bilateral breast metastases from ewing sarcoma of the femur. Am J Clin Oncol. 2005;28(1):102103.

4. Chuthapisith S, Prasert W, Warnnissorn M. Ewing's sarcoma and primitive neuroectodermal tumour (ES/PNET) presenting as a breast mass. Oncol Lett. 2012;4(1):67-70.

5. Orguc S, Basara I, Pocan T, et al. Ewing's sarcoma metastasis into the breast. Diagn Interv Radiol. 2012;18(2):167-70.

6. Desai SS, Jambhekar NA. Pathology of Ewing's sarcoma/PNET: Current opinion and emerging concepts. Ind J Orth. 2010;44(4):363-368.

7. Sunita S, Shilpa G, Monika S, Jyoti S, et al. Ewing's sarcoma humerus presenting along with metastatic breast nodule: An unusual case report. Clin Cancer Inv J. 2013;2(4):368-370.

8. Tot T, Badani DE, La Parra JJ, et al. Metaplastic carcinoma of the breast with neuroectodermal stromal component. Pathol Res Int. 2011;20(1):19-24.

9. Abdull Gaffar B, Ghazi E, Mohamed E, et al. Breast metaplastic carcinoma with unusual small cell component. Breast Dis. 2012;34(1):19-24.

10. Ijichi K, Tsuzuki T, Adachi M, et al. A peripheral primitive neuroectodermal tumor in the larynx: A case report and literature review. Oncology Lett. 2016;11(2):1120-1124.

11. Milanezi F, Pereira EM, Ferreira FV. CD99/MIC-2 surface protein expression in breast carcinomas. Histopathol. 2001;39(6):578-583.

12. Friedrichs N, Vorreuther R, Poremba C. Primitive neuroectodermal tumor (PNET) in the differential diagnosis of malignant kidney tumors. Pathol Res Pract. 2002;198(8):563-569.

13. Lee SH, Park JM, Kook SH, et al. Metastatic tumors to the breast: Mammographic and ultrasonographic findings. $J$ Ultrasound Med. 2000;19(4):257-262.

14. Peersman B, Vanhoenacker FM, Heyman S, et al. Ewing's sarcoma: Imaging features. JBR-BTR. 2007;90(5):368-376. 
15. Pawar VR, More S, Patil PP. Cytological diagnosis of Ewing sarcoma. Ind Med Gaz. 2011;12:495-497.

16. Jain BB, Choudhury CR, Bhutia TD, et al. Metachronous presentation of breast sarcoma following carcinoma: A rare occurrence. Clin Cancer Invest J. 2013;2(4):359-361.

17. Grier HE, Krailo MD, Tarbell NJ. Addition of ifosfamide and etoposide to standard chemotherapy for Ewing's sarcoma and primitive neuroectodermal tumor of bone. The New England Journal of Medicine. 2003;348(8):694-701.
18. Chung SY, Oh KK. Imaging findings of metastatic disease to the breast. Yonsei Med J. 2011;42(5):497-502.

19. Majid N, Amrani M, Ghissassi I, et al. Bilateral ewing sarcoma/primitive neuroectodermal tumor of the breast: A very rare entity and review of the literature. Case Reports Oncol Med. 2013;13(3):12-14.

20. Tamura G, Sasou S, Kudoh S. Primitive neuroectodermal tumor of the breast: Immunohistochemistry and fluorescence in situ hybridization. Pathol Int. 2007;57(8):509-512. 